\title{
Effective Analysis of Lung Infection using Fuzzy Rules
}

\author{
Sharad Kumar Tiwari ${ }^{1}$, Navneet Walia ${ }^{2}$, Harsukhpreet Singh ${ }^{3}$ and Anurag Sharma ${ }^{4}$ \\ 2, 3, 4 CT Institute of Technology and Research, Jalandhar \\ ${ }^{1}$ Thapar University, Patiala \\ navneetwalia356@gmail.com
}

\begin{abstract}
Soft Computing is a consortium of methodologies which works synergistically and provides an ability to make decision from reliable data or expert's experience. Nowadays different types of soft computing techniques such as neural network, fuzzy logic, genetic algorithm and hybrid system are largely used in medical areas. In this paper, algorithm for analysis of lung infection is presented. The main focus is to develop system architecture to find probable disease stage patient may have. Severity level of disease is determined by using rule base method. The algorithm uses output of Rulebase and entered by user to determine level of infection.
\end{abstract}

Keywords: Decision support system, Fuzzy logic, Rulebase diagnosis

\section{Introduction}

Medical diagnosis is one of the most important issues in healthcare. The medical industry requires new engineering technologies, to assess information objectively. While recent developments in medical engineering have been achieved by state-of-the-art of intelligent computing techniques including computer-aided diagnosis, computer-aided radiography, developments in computational techniques including soft computing (SC), information processing and data mining hold new premises in this field. SC methods are becoming indispensable for to sport modern medical practice [1]. "Soft computing differs from conventional (hard) computing in that, unlike hard computing, it is tolerant of imprecision, uncertainty, and partial truth. In effect, the role model for soft computing is the human mind. The guiding principle of soft computing is: exploit the tolerance for imprecision, uncertainty, and partial truth to achieve tractability, robustness and low solution cost" described by Prof. Lofti Zahed [2]. The accuracy of the diagnosis contributes into the right treatment and subsequently the cure of the disease. In recent years, computational intelligence has been widely used to solve many complex problems because of its capability to handle tolerance for imprecision, uncertainty, approximate reasoning and partial truth. Soft computing is a combination of fuzzy logics (FL), neural network (NN) and genetic algorithms (GA) methodologies. All these methodologies works synergistically and provides, in one form or another, flexible information processing capability for decision making systems, such as expert systems and pattern classification system [3]. In real world computing environment, information is not complete, full of uncertainty and imprecise which makes difficult to derive actual decision. Various decision support systems have been constructed with the help of artificial intelligence. These systems have widely proved very useful for medical experts in making prompt decision about diagnosis [4]. In advent of technology, computerization in health care unit allows various clinical decision making unit to be constructed; that can performs computation as human expert in narrow domain problem. Clinical decision support 
systems are broadly classified into two main groups as shown in Figure 1. (i) Knowledge based Clinical Decision Support System (ii) Non-Knowledge based Clinical Decision Support System

\subsection{Knowledge based Clinical Decision Support System:}

Knowledge based clinical system contains a set of If-then rules that are used to describe a certain patterns. Observed data are collected and used to evaluate these rules. If the rules are logically satisfied, the pattern is identified and problem associated with that pattern is evaluated. For example if pain intensity exceeded upto certain level then warning pattern is generated. Each particular problem employs a specific treatment. The knowledge based clinical decision support system consists of three main parts: Knowledge base component, Inference engine, Fuzzification component. Knowledge base unit contains rulebase and database that defines the membership functions of fuzzy sets used in fuzzy rules. Inference unit performs operation on the rules to convert crisp quantity into fuzzy quantities. Fuzzification unit use to translate input values into fuzzified values [5].

\subsection{Non-Knowledge based Clinical Decision Support System}

Clinical decision support systems without knowledge base unit are called non knowledge based clinical decision support system. These systems used a form of artificial intelligence called as machine learning. This system is divided into two types as mentioned below:

\section{A. Artificial Neural Network (ANN)}

A neural network is a data processing system consisting of large number of simple, highly interconnected processing elements (artificial neurons) inspired by neuroscience or neurobiology. It resembles human brain in two respects, (i) knowledge is acquired by network from its environment through a learning process, (ii) interneuron connection strengths, known as synaptic weights, are used to store the acquired knowledge. It is a system with input and outputs composed of many simple and similar processing elements. The procedure used to perform the learning process is called learning algorithm, the function of which is to modify synaptic weights of the network in an orderly fashion to attain desired design objective. Neural network are robust systems having capability of fault tolerance and exhibit learning ability to map input patterns to associate output patterns based on limited set of data $[6,7]$. Neural network use nodes and weighted connections to derive relationship between symptoms and diagnosis.

\section{B. Genetic Algorithm (GA)}

Genetic algorithm are subclass of evolutionary algorithm (EA's) that use biology inspired mechanism, where elements of search space are binary strings (chromosomes) which correspond to a particular solution. GA is computer based global search technique effective in acquiring optimal solution for solving optimization problems by simulating the mechanisms of genetics and natural selection. The major weakness of GA is that it usually tends to be (i) computationally expensive in real systems, (ii) premature convergence and (iii) slow search speed. This method is appropriate when we donot need to find best solution, only appropriate is required [8]. Selection algorithm evaluates components of solution to complex problem. The generic system goes through number of iterative methods to evaluate best solution of 
required problem. The parameter optimization approach has been widely adopted in number of genetic fuzzy systems [9].

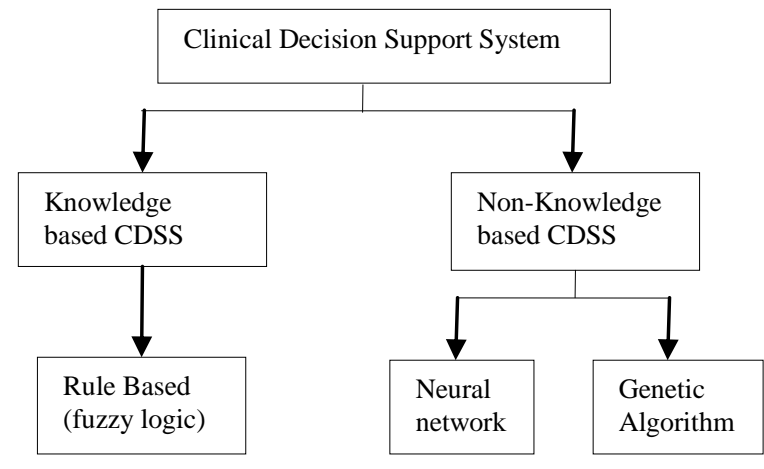

Figure 1. Types of Clinical Decision Support System

Fuzzy set theory has a number of properties that make it suitable for formalizing the uncertain infonnation upon which medical diagnosis and treatment is usually based. Firstly, it defines inexact medical entities as fuzzy sets based on membership rather than classical binary logic. Secondly, it provides a linguistic approach with an excellent approximation to texts. It is powerful tool to tackle imprecision and uncertainty. Finally, fuzzy logic offers reasoning methods capable of drawing approximate inferences. Fuzzy set theory is aid in providing symbolic knowledge information in more human comprehensible or natural form, and can handle suspicions at various levels. These facts suggest that fuzzy set theory can be employed for the development of a computerized diagnosis system [10,11]. Since fuzzy logic resembles human decision making with its ability to work from approximate reasoning and ultimately to find precise solution, it can be applied in the diagnosing and monitoring of diseases including lung infections.

The goal is to mimic the actions of a domain expert who can solve complex problems. Fuzzy logic has proved wonderful tool for decision making systems and it is appropriate if sufficient expert knowledge about the process is available [9]. In the present paper, fuzzy rule base system is designed to serve a decision support for predicting lung infection. Lung diseases occurs mainly through contagious bacterial infections and involves lungs, but may spread to other organs. The person gets infected by breathing in air droplets from cough or sneeze of an infected person. Common symptoms involves breathing difficulty, cough, haemoptysis are the common indications of lung infections. The scope of this work is to develop prototype warning system for clinical activity, based on assumption that clinical problems can be analysed using simple rules and decision process of physician can be modelled by using sets of fuzzy rules. Rule based systems are mainly used in medical diagnosis. In this, the knowledge base stores all information about the symptoms and disease in the form of rules in the Rule base [12]. Fuzzy logic provides inference morphology to build a system based on a set of user supplied human language rules. However, fuzzy logic is highly abstract and converts these rules to their mathematical equivalents. This simplifies the job of the system designer and the computer, and results in much more accurate representation of the way systems behave in the real world $[13,3]$. 


\section{Review of Related Work}

In this section, we will introduce some related works in the field of fuzzy logic. A detailed survey of fuzzy logic techniques is found in this section. There are many works in literature that explains design and implementation of medical experts system.

A computational system based on neurofuzzy logic for identification of Alzheimer was presented by J.C. Obi, et al., (2011) [15]. This system uses a set of fuzzified dataset symptoms into neural system to produce more precise output than traditional method. The implementation results reveal that hybrid system is introduced in healthcare delivery centers to help ease the work of physicians. To manage uncertainty and imprecision in measurements related to size, shape of abnormality, fuzzy inference system was designed for assessment of peptic ulcers by K. Rezai, et al., (2014) [16]. The results of proposed approach models vagueness and uncertainty associated to the measurements of small objects in low resolution images. The encouraging results of intelligent fuzzy inference system can help medical experts as second reader for detection of peptic ulcer in decision making process and consequently, improves treatment process. Experimentally it is shown that intelligent system provides maximum accuracy of $98.1 \%$, which reveals superiority of the approach.

Fuzzy logic can be applied to determine and identify lung cancer by designing fuzzy rule based inference system was discussed by K. Lavanya, et al., (2011) [17]. The proposed system accepts the required symptoms for lung infection and calculates the confirmed disease stage as output. Based on the inputs provided, system calculates the membership function for both inputs and outputs variables. This approach requires expert knowledge gathered to generate rules and rules are fired accordingly to generate symptoms. The implementation results were carried out in JADE accordingly to generate appropriate symptoms. The implementation results were carried out in JADE and MATLAB using features of fuzzy logic toolbox. An attempt has also been implemented for providing treatment to patients. Further, it was generated that this system is compatible with other techniques and can be easily implemented using other methods such as case based reasoning, decision tree and data mining methods. System efficiency can be increased by making use of neurofuzzy system and rough sets. O. Opeyemi, et al., (2012) suggested a novel approach for early prediction of heart attack using neuro-fuzzy system. The neuro fuzzy system was designed using eight input variables and one output variable [18]. Initially dataset used was extracted from database, and then the initial fuzzy logic system was generated. The network was trained with set of trained data and validation is performed. An output of system was designed in such a way that patient can use it personally. Based on the values (symptoms provided by patient) supplied, system will able to predict risk level of patient. The result of proposed designed system achieves accuracy $90 \%$ and it is inferred from results that neuro-fuzzy model is suitable and feasible to be used as a supportive tool for disease diagnosis.

Usefulness of fuzzy-neural algorithm techniques for prediction of various diseases as well as to determine critical stage of patients in medical diagnosis can be used to provide timely remedial actions and it can prevent a major damage to health of patients, was demonstrated by K. Ketal, et al., (2014) [20]. Purpose of this study is to evaluate increasing usage of fuzzyneural network and FPGA based implementation can be used as an expert system for predicting various physiological diseases. Design of various fuzzy neural algorithms was presented such as back propagation, inverse delayed function and time averaging types are frequently to predict level of various diseases. An attempt was made to explain why smart type prediction systems such as FPGA, mostly used by doctors to provide a complete smart solution for the prediction of any disease. An integrated approach for glaucoma detection using adaptive neuro fuzzy inference system was carried out by M. Huang, et al., (2007) [19]. 
The purpose of this work was to develop an automated classifier based on adaptive neuro fuzzy inference system, to differentiate between normal and glaucomatous eyes from the quantitative evaluation of summary data reports of the Stratus optical coherence tomography (OCT) in Taiwan Chinese population. In this study decision making was performed in two stages; features are selected using orthogonal array and then training is performed using backpropagation gradient method in combination with least square method. It is investigated that with stratus OCT parameter as input, ANFIS generates better results for discriminating between glaucomatous and normal eyes. Therefore, use of adaptive neuro fuzzy inference system is preferable since output concludes the if-then rules and membership functions, which enhances the readability of the output. A survey on application of fuzzy logic in medical diagnosis was performed by V. Prasath, et al., (2013) [4]. author surveyed that various clinical decision support system have been constructed via aid of artificial intelligence and are widely accepted in hospitals, clinics for making decisions. This system seems to be designed in such a way that they have ability to think logically and to deal with approximate reasoning. This paper presents application of fuzzy logic in medical field such as tuberculosis, cancer, image and signal processing, aphasia, pharmacy, heart diseases, asthma, diabetes, malaria, hypothyroidism, HIV, arthritis, anesthesia and menigioma. It is concluded that fuzzy logic provides a means for encapsulating the subjective decision making process suitable for computer implementation.

Soft computing is a combination of methodologies which works synergistically and provides flexible information processing potential for handling uncertain situations. A. Yardimci (2009) surveyed on various soft computing techniques in medicinal field and aims to explain briefly about various soft computing methodologies between years 2000 and 2008 [1]. In this paper research work research work on MEDLINE was explained. Basic study involves the current elements of soft computing, different mechanisms implemented in soft computing techniques and to establish a guide which help to forecast the future developments of soft computing in medicine. Further, use of soft computing methods in wide range of fields such as imaging, classification, diagnosis, prediction and control in medicine was suggested. A rule based fuzzy diagnostics decision support system for tuberculosis was proposed by A. Semaogan (2011) [21]. The proposed medical support system was specialized for pulmonary physicians focusing on tuberculosis. The main objective of study is to develop system architecture and algorithm to find class of tuberculosis patient may have. The develop decision support system would assist pulmonary physicians to diagnose ailment, and to generate probable class of tuberculosis determined by using a rule base system. This developed system would greatly helps in decision making and lessens the time consumed in generating diagnosis. A fuzzy framework for diagnosis and monitoring of cholera is presented by A.Udak (2013) for providing decision support platform to cholera health practioners [23]. The motivation of the work was due to insufficient cholera control measures in existence and need to provide novel approach towards cholera control. In this work fuzzy expert system consists of four components, includes; knowledgebase, fuzzification, inference engine, defuzzification. The medical expert inference engine employ mamdani max-min fuzzy expert system and centriod of area defuzzification technique to infer data from rules developed. The medical expert system is useful for medical practioners and healthcare sector in making prompt decision during diagnosis of cholera.

Data mining solution for diagnosis of tuberculosis by T. Ucar, et al., (2013) was developed for providing decision support to tuberculosis researchers [14]. The purposed technique used adaptive neuro fuzzy inference system and rough sets that makes diagnosis of tuberculosis as accurate as possible. This approach helps in deciding whether it is reasonable to start treatment on suspected patients without waiting for exact medical test results. Experimentally, 
it is shown that use of Sugeno-type ANFIS classifies the instances with correctness of 97\%, whereas rough sets does same classification with correctness of $92 \%$.

It is inferred from above literature survey on fuzzy logic that they are successfully applied in many medical fields for diagnosing and monitoring of various diseases. In this work fuzzy rules are applied to determine probability of lung infection.

\section{Fuzzy Diagnostic Decision Support System}

\subsection{Proposed Fuzzy Medical Diagnosis System}

This section describes the approach adopted in developing the overall framework for medical diagnosis system of lung infection. The framework comprises of three main components: knowledge based, fuzzy inference system and defuzzified value as shown in Figure 2. This system will offer potential assistance to medical practioners and health care sector in making prompt decision of lung infection.

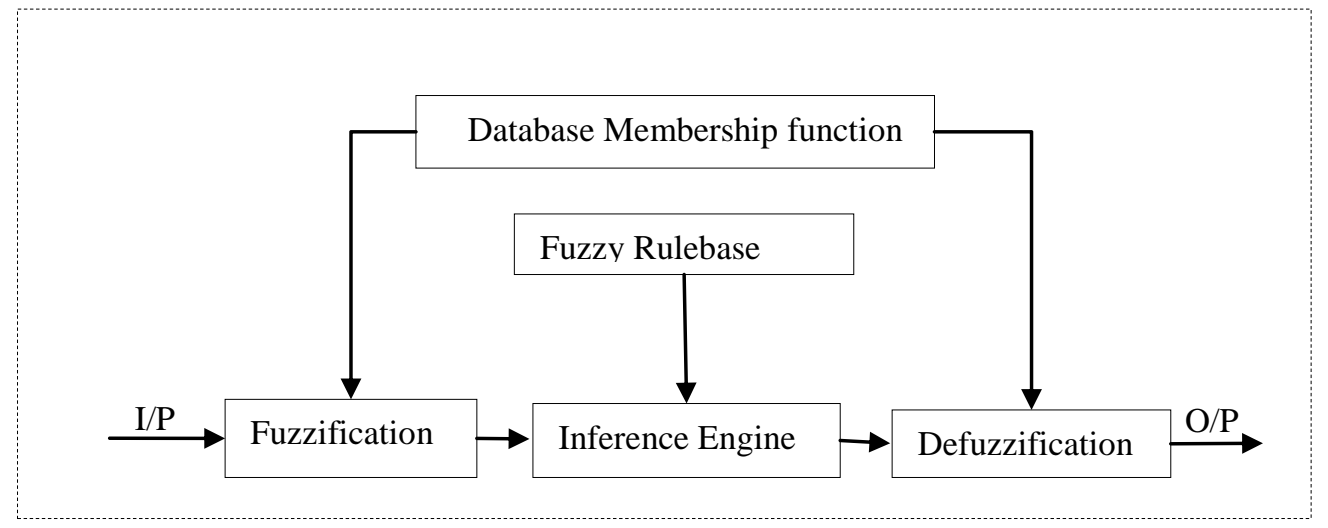

Figure 2. Framework of Fuzzy Expert System

\subsubsection{Identification Parameter}

It is essential to take into consideration of all symptoms that plays significant role in causing lung infection [23]. Take these aspects into consideration for purpose of this study, a set of input and output parameters taken into account have been listed in Table 1.

Table 1. Parameters and its Corresponding Functions

\begin{tabular}{|l|l|l|}
\hline \multirow{3}{*}{ Type (input/ output) } & Parameter & Membership values \\
\hline Cough & $\begin{array}{l}\text { Low using trapmf, } \\
\text { Medium using trimf } \\
\text { High using trapmf }\end{array}$ \\
\cline { 2 - 3 } & Haemoptysis & $\begin{array}{l}\text { Low using trimf } \\
\text { Medium using trapmf } \\
\text { High using trimf }\end{array}$ \\
\hline Output & $\begin{array}{l}\text { Probability of } \\
\text { lung infection }\end{array}$ & $\begin{array}{l}\text { Severity of disease } \\
\text { represented on scale 1-10 }\end{array}$ \\
\hline
\end{tabular}

\subsubsection{Choice of Membership Function}


Membership functions are chosen for each values of input parameter. Selection of appropriate membership value for each and every value of input and output plays vital role in mapping efficiency of system. The degree an input belongs to a fuzzy set is denoted by membership function between 0 and 1. Apart from these the number of membership functions, a number of other correspondence factor need to be taken into account. These factors includes the type, parameter, aggregation operator (AND, OR), inference operator, type of fuzzification and defuzzification used. On observation of behavioural characteristics of each fuzzy variable encountered in problem is represented by using triangular and trapezoidal membership function as shown in Figure 3 and Figure 4.

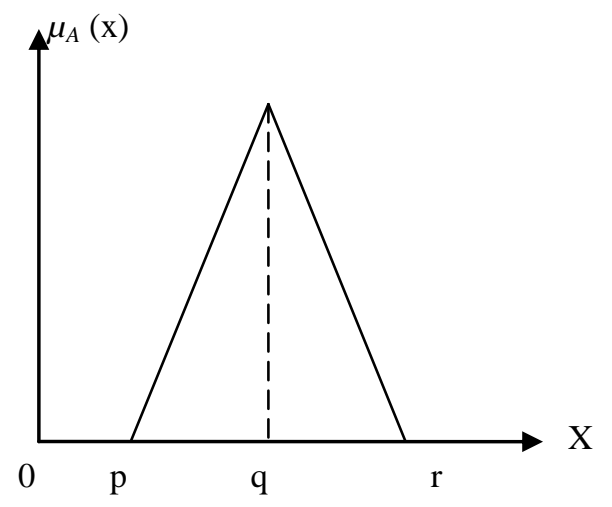

\section{Figure 3. Triangular Membership Function}

A membership function associated with given fuzzy set maps an input value to its appropriate membership value and its value must be in $[0,1]$. Fuzzy set $\mathrm{A}$ in $\mathrm{X}$ is defined as set of ordered pairs:

$$
\mathrm{A}=\left\{\mathrm{x}, \mu_{\mathrm{A}}(\mathrm{x}), \quad \mathrm{x} \in X\right\}
$$

$\mu_{\mathrm{A}}(\mathrm{x})$, is called membership function of $\mathrm{x}$ in A. fuzzy set is a set without a crisp value, clearly defined boundary. It contains elements with only partial degree of membership. The triangular membership function curve is function of three variables $p, q, r$ in $x$-axis where $p, r$ are "feet values" respectively, having membership degree zero and q is the "peak value" whose membership degree is one. Triangular membership is represented in equation 1.

$$
\mathrm{f}(\mathrm{x}, \mathrm{p}, \mathrm{q}, \mathrm{r})=\left\{\begin{array}{cl}
0 & \text { if } \mathrm{x}<=\mathrm{p} \\
\frac{x-p}{q-p} & \text { if } p<x \leq q \\
\frac{r-p}{r-q} & \text { if } \mathrm{q}<=x<r \\
0 & \text { if } \mathrm{x}>=\mathrm{r}
\end{array}\right\}
$$

Trapezoidal membership function is represented by using four variables $\mathrm{p}, \mathrm{q}, \mathrm{r}$ and $\mathrm{s}$ in $\mathrm{x}$ axis where $\mathrm{p}$ and $\mathrm{s}$ locate "feet" of trapezoid whose membership degree is zero, parameter $\mathrm{q}$ and $r$ locate the "shoulders" whose membership is one represented in equation 2 [23]. 


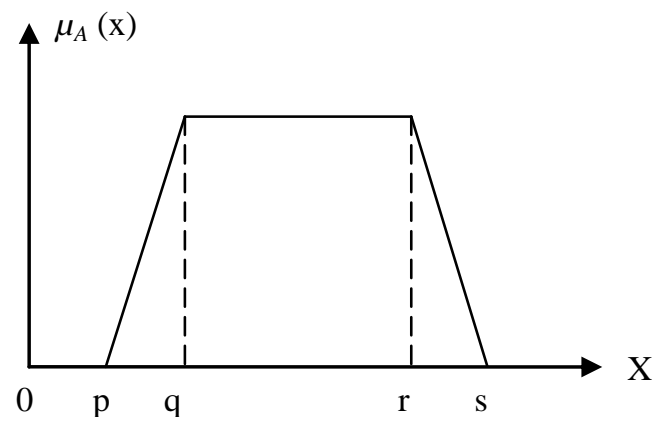

Figure 4. Trapezoidal Membership Function

Gaussian, Sigmoid and other types of linear functions can also be useful to distinguish the fuzzy sets. Non-linear functions can also be used but they will further cause computational complexity to the algorithm

$f(x, p, q, r, s)=\left\{\begin{array}{cl}0 & \text { if } x<=p \\ \frac{x-p}{q-p} & \text { if } p \leq x \leq q \\ 1 & \text { if } q<=x<=r \\ \frac{s-x}{s-r} & \text { if } r \leq x \leq s \\ 0 & \text { if } s<=x\end{array}\right\}$

\section{System Modelling and Working}

Fuzzy logic was the tool used to develop the algorithm of system for determining the actual output. By using fuzzy, researcher will able to classify intensity of each symptom according to description prescribed by pulmonary physicians. This paper is a simulation based paper and medical diagnosis system is performed on fuzzy logic model by using MATLAB 2013b software package developed by Mathworks Inc. Figure 3 shows the command window of MATLAB software where we use write commands to operate given set of rules while programming [24].

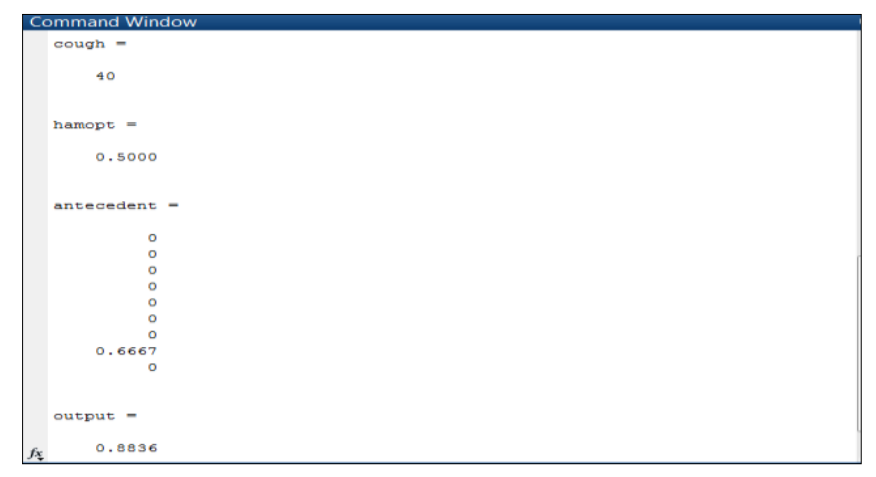

Figure 5. Command Window View

Rule base method is designed and implemented to determine probability of lung infection populated by rules made for above inputs. Fuzzy logic employs the output of rule-based 
method and calculates range of scores in order to determine final output. Defuzzification process is used to formulate the rules that correspond to different conditions determined by matrix. Symptoms used to determine probability of lung infection like cough, haemoptysis were classified as low risk, medium risk and high risk. In formulation of fuzzy sets, the ranges of input and output variable symptoms are identified and corresponding range of meaningful linguistic states are selected by expressing with appropriate fuzzy logic for each symptom. Figure 6 shows fine tuned membership function plot for cough showing number of day's patient suffering from cough.

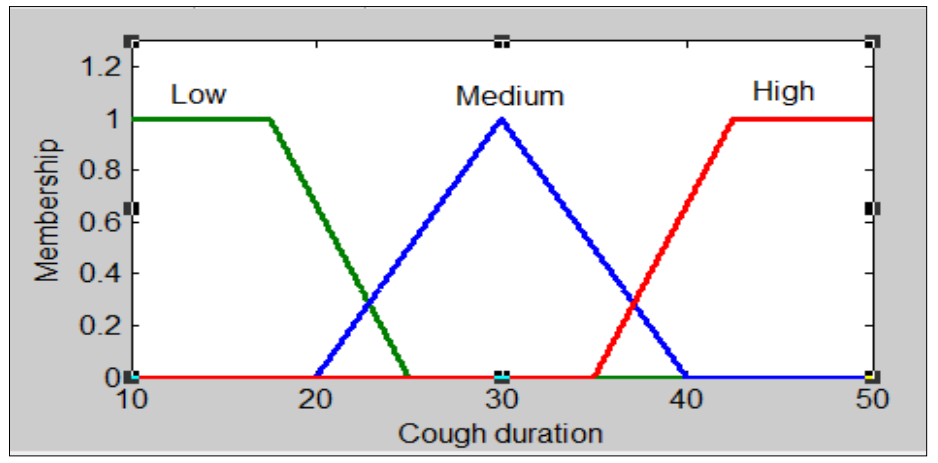

Figure 6. Fine Tuned Membership Plot for Cough

The proposed system requires the physician to input all necessary scores of symptoms needed in order to meet the required output. System input symptom scores with corresponding level of intensity as follows:

Cough duration [10 50], Haemoptysis [0 1].

A sample choice of membership function and its values has been shown below:

$$
\begin{aligned}
& \text { Name }=\text { 'Cough' } \\
& \text { Range }=\left[\begin{array}{ll}
10 & 50
\end{array}\right] \\
& \text { NumMFs }=3 \\
& \text { MF1 = 'Low': 'trapmf', [10 } 1017.5 \text { 25 }] \\
& \text { MF2 = 'Med': 'trimf', [20 } 30 \text { 40] } \\
& \text { MF3 = 'High': 'trapmf', [35 } 42.550 \text { 50] }
\end{aligned}
$$

Figure 7 shows fined tuned fuzzy relations of haemoptysis showing membership to different categories (low, med, high). A sample choice of membership function and its values for haemoptysis has been shown below:

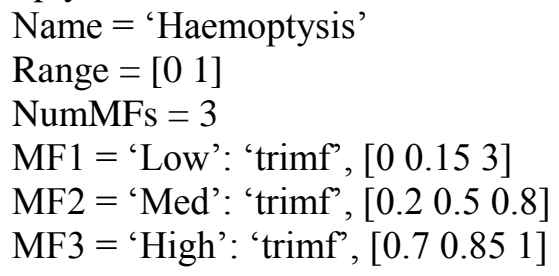




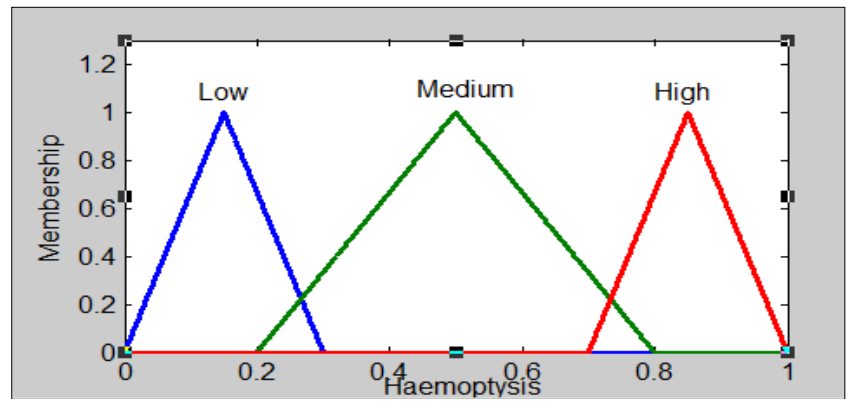

Figure 7. Fine Tuned Membership Plot for Haemoptysis

After the graph, fuzzy sets were then intersected and it is determines the matrix. Two symptoms used are intersected and it describes one output condition. The matrix illustrates intersection between two symptoms. For evaluation of rules, the input scores had undergone defuzzification process. After defuzzification, the scores were subjected to determine value of crisp output. Below is a sample illustration of input and output scores:

Antecedent rules: antecedent $1=\min ($ coughlow_mf $($ find $(\mathrm{x}==\mathrm{cough}))$, hamlow_mf (find $(\mathrm{y}==$ hamopt $)))$; antecedent $2=\min ($ coughlow_mf(find $(x==$ cough $))$,hammed_mf (find $(y==$ hamopt $))$ ); antecedent $3=\min ($ coughlow_mf(find $(\mathrm{x}==$ cough $))$,hamhigh_mf (find $(\mathrm{y}==$ hamopt $)))$; antecedent $4=\min ($ coughmed_mf(find $(\mathrm{x}==\mathrm{cough}))$, hamlow_mf (find $(\mathrm{y}==$ hamopt $))$ ); antecedent $5=\min ($ coughmed_mf $($ find $(\mathrm{x}==\mathrm{cough}))$,hammed_mf (find $(\mathrm{y}==\mathrm{hamopt})))$; antecedent $6=\min ($ coughmed_mf $($ find $(\mathrm{x}==\mathrm{cough}))$, hamhigh_mf (find $(\mathrm{y}==$ hamopt $)))$; antecedent $7=\min ($ coughhigh_mf $($ find $(\mathrm{x}==\mathrm{cough}))$,hamlow_mf (find $(\mathrm{y}==$ hamopt $)))$; antecedent $8=\min ($ coughhigh_mf $($ find $(\mathrm{x}==\mathrm{cough}))$,hammed_mf (find $(\mathrm{y}==$ hamopt $)))$; antecedent $9=\min ($ coughhigh_mf $($ find $(\mathrm{x}==\mathrm{cough}))$,hamhigh_mf $($ find $(\mathrm{y}==$ hamopt $)))$;

Consequent rules:

consequent 1 = infelow_mf. $*$ antecedent 1 ;

consequent $2=$ infelow_mf. $*$ antecedent 2 ;

consequent $3=$ infemedlow_mf. $*$ antecedent 3 ;

consequent $4=$ infemedlow_mf.* antecedent 4 ;

consequent $5=$ infemed_mf. $*$ antecedent 5 ;

consequent 6 = infemedhigh_mf. * antecedent6;

consequent $7=$ infemedhigh_mf. $*$ antecedent 7 ;

consequent $8=$ infehigh_mf.*antecedent 8 ;

consequent 9 = infehigh_mf.*antecedent 9 ;

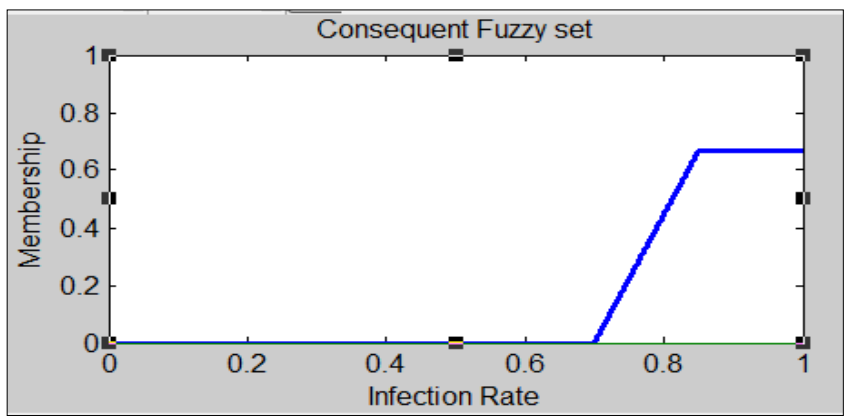

Figure 8. Consequent Rule view 
Figure 8 illustrates consequent fuzzy set for value of haemoptysis 0.5 and cough 40 . Upon entering values of cough as 40 and haemoptysis as 0.5 , system generates output 0.8836 which corresponds that there is probability of lung infection. When we type values in editor window then system corresponds to defuzzify a value which is shown in Figure 9.

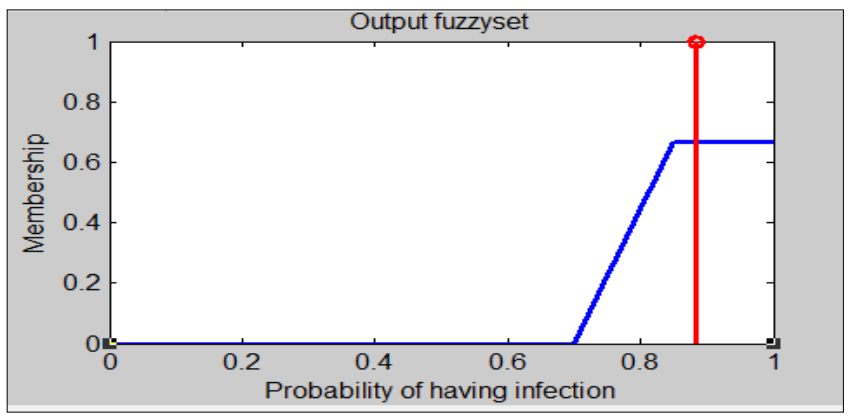

Figure 9. Defuzzified Value

\section{Conclusion}

In this paper, we have investigated probability of occurrence of lung infection using random number of inputs for lung management system. The design of fuzzy logic in design of such automated system gives an innovative and professional means of lung infection diagnosis especially in areas where infection is in wide spread. In this paper we formalize medical entities as fuzzy sets and formalize reasoning using rule based systems. The use of fuzzy logic in design of diagnostic system proposed in this paper is believed to serve as a dependable and cheap means of treating lung diseases. Furthermore, implementation of fuzzy logic provides a set of techniques generating reliable solution.

\section{References}

[1] A. Yardimci,“Applications of soft computing to medical problems,” International Conference on Intelligent Systems Design and Applications, (2009), DOI: 10.1109/ISDA.2009.168.

[2] J. E. Maiers, "Fuzzy set theory and medicine," IEEE trans., (1985),pp. 325-329.

[3] K. Soundararajan, "Diagnostics Decision Support System for Tuberculosis using Fuzzy Logic," International Journal of Computer Science and Information Technology \& Security, ISSN: 2249-9555 vol. 2, no. 3, (2012), pp. 684-689.

[4] V. Prasath, N. Lakshmi, M. Nathiya, N. Bharathan and N. P. Neetha "A Survey on the Applications of Fuzzy Logic in Medical Diagnosis Support Systems Systems Decision," International Journal of Scientific \& Engineering Research, ISSN 2229-5518, vol. 4, no. 4, (2013), pp. 1199-1203.

[5] X. Y. Djam and Y. H. Kimbi, "A Decision Support System for Tuberculosis Diagnosis," The Pacific Journal of Science and Technology, vol. 12, no. 2, (2011), pp. 410-425.

[6] S. P. Singh and P. Johri, "A Review of Estimating Development Time and Efforts of Software Projects by Using Neural Network and Fuzzy Logic in MATLAB," International Journal of Advanced Research in Computer Science and Software Engineering, vol. 2, no. 10, (2012), pp. 306-310.

[7] P. D. C. R. Jayarathna, J. V Wijayakulasooriya and S. R. Kodituwakku, "Fuzzy Logic and Neural Network Control Systems for Backing up a Truck and a Trailer,” Int. J Latest Trends Computing, ISSN: 2045-5364, no. 3, (2011), pp. 370-377.

[8] R. Malhotra, N. Singh and Y. Singh, "Genetic Algorithms : Concepts, Design for Optimization of Process Controllers," Canadian Center of Science and Education, vol. 4, no. 2, (2011), pp. 39-54.

[9] O. Taylan, "Computers \& Industrial Engineering An adaptive neuro-fuzzy model for prediction of student 's academic performance", Computers \& Industrial Engineering, Elsevier, doi:10.1016/j.cie.2009.01.019, vol. 57, (2009), pp. 732-741.

[10] K. Adlassnig, "Fuzzy Set Theory in Medical Diagnosis," IEEE Transactions on Systems, Man, and Cybernetics, (1986), pp. 260-265. 
[11] N. Walia, S. K. Tiwari and R. Malhotra, "Design and Identification of Tuberculosis using Fuzzy Based Decision Support System,” Advances in Computer Science and Information Technology, ISSN: 2393-9915, vol. 2, no. 8, (2015), pp. 57-62.

[12] M. A. S. Durai, N. C. S. N. Iyengar and A. Kannan, "Enhanced Fuzzy Rule Based Diagnostic Model for Lung Cancer using Priority Values," International Journal of Compputer science and information technologies, vol. 2, no. 2, (2011), pp. 707-710.

[13] N. Annals and O. F. Natural, "Decision Support System fot the Identification of Tuberculosis using Neuro Fuzzy Logic", Nigerian Annals of Natural Sciences, vol. 12, no. 1, (2012), pp. 12-20.

[14] T. Uc, A. Karahoca and D. Karahoca, "Tuberculosis disease diagnosis by using adaptive neuro fuzzy inference system and rough sets," Neural Comput \& Applic, DOI 10.1007/s00521-012-0942-1, (2013), pp. 471-483.

[15] J. C. Obi and A. A. Imainvan, "Decision Support System for the Intelligient Identification of Alzheimer using Neuro Fuzzy logic,” International Journal on Soft Computing, DOI : 10.5121/ijsc.2011.2203, vol. 2, no. 2, (2011), pp. 25-38.

[16] K. Rezaei, R. Hosseini and M. Mazinani, "A Fuzzy Inference System For Assessment of The Severity of The Peptic Ulcers,” DOI : 10.5121/csit.2014.4527, (2014), pp. 263-271.

[17] K. Lavanya, M. A. S. Durai, N. C. Sriman and N. Iyengar, "Fuzzy Rule Based Inference System for Detection and Diagnosis of Lung Cancer," International Journal of Latest Trends in Computing ISSN: 2045-5364, vol. 2, (2011), pp. 165-171.

[18] O. Opeyemi, "Development of Neuro-fuzzy System for Early Prediction of Heart Attack," Information Technology and Computer Science, DOI: 10.5815/ijitcs.2012.09.03, (2012), pp. 22-28.

[19] M. Huang, "Glaucoma detection using adaptive neuro-fuzzy inference system," Expert Systems with Applications, Elsevier, doi:10.1016/j.eswa.2005.12.010, vol. 32, (2007), pp. 458-468.

[20] K. Ketan, "Applications of Fuzzy-Neural and FPGA for Prediction of Various Diseases- A survey," International Journal of Advanced Research in Electrical, Electronics and Instrumentation Engineering, ISSN: 2278- 8875, (2014), pp. 13588-13593.

[21] A. R. C. Semogan, B. D. Gerardo, "A Rule-Based Fuzzy Diagnostics Decision Support System for Tuberculosis," International Conference on Software Engineering Research, Management and Applications, DOI 10.1109/SERA.2011.30, (2011).

[22] A. Uduak and M. Mfon, "A Proposed Fuzzy Framework for Cholera Diagnosis and Monitoring," International Journal of Computer Applications, vol. 82, no. 17, (2013), pp. 1-10.

[23] S. K. Anand, R. Kalpana, S. Vijayalakshmi, S. Hartley and B. Boucho-meunier, "Design and Implementation of a Fuzzy Expert System for Detecting and Estimating the Level of Asthma and Chronic Obstructive Pulmonary Disease," World Applied Sciences Journal, DOI: 10.5829/idosi.wasj.2013.23.02.13046, vol. 23, no. 2, (2013), pp. 213-223.

[24] D. Kumar, S. Parkash, P. K. Bhatia and H. Kaur, "Fuzzy Based Temperature Controller Using Membership Functions In Fuzzy Toolbox," Second International Conference on Advances in Computing and Communication Engineering, DOI 10.1109/ICACCE.2015.90, (2015).

[25] E. Victor, O. Uyinomen, E. Enobakhare and A. Emmanuel, "Full Length Research Paper A fuzzy inference system for predicting depression risk levels," African Journal of Mathematics and Computer Science Research, DOI: 10.5897/AJMCSR2013.0511, ISSN 2006-9731, vol. 6, no. 10, (2013), pp. 197-204.

[26] I. Gu, "Application of adaptive neuro-fuzzy inference system for detection of electrocardiographic changes in patients with partial epilepsy using feature extraction," Expert Systems with Applications , Elsevier, vol. 27, (2004), pp. 323-330.

[27] E. D. Ubeyli, "Adaptive neuro-fuzzy inference system for classification of EEG signals using wavelet coefficients," Journal of Neuroscience Methods, Elsevier, doi:10.1016/j.jneumeth.2005.04.013, (2005).

[28] J. B. Awotunde, O. E. Matiluko and O. W. Fatai, "Medical Diagnosis System Using Fuzzy Logic," African Journal of Computing \& ICT, vol. 7, no. 2, (2014), pp. 0-7.

[29] A. Kaur, "Development of Constant Sugeno Type Fuzzy Inference System for Load Sensor," International Journal of Engineering Science and Innovative Technology, ISSN: 2319-5967, vol. 2, no. 2, (2013), pp. 564567.

[30] P. H. B. Ierling and E. Epage, "A UMLS-based Knowledge Acquisition Tool for Rule-based Clinical Decision Support System Development," Journal of the American Medical Informatics Association, vol. 8, no. 4, (2001), pp. 351-360.

[31] P. A. Adewuyi, "Performance Evaluation of Mamdani-type and Sugeno-type Fuzzy Inference System Based Controllers for Computer Fan", Information Technology and Computer Science, DOI: 10.5815/ijitcs.2013.01.03, (2013), pp. 26-36. 